\title{
Energy Aware Metaheuristic Optimization with Location Aided Routing Protocol for MANET
}

\author{
E. Ahila Devi' ${ }^{1}$, K. C. Ramya², K. Sathesh Kumar ${ }^{3}$, Sultan Ahmad ${ }^{4}$, Seifedine Kadry ${ }^{5}$, Hyung Ju Park \\ and Byeong-Gwon Kang ${ }^{6, *}$
}

\author{
${ }^{1}$ Department of Instrumentation and Control Engineering, St. Joseph's College of Engineering, Chennai, 600119, India \\ ${ }^{2}$ Department of Electrical and Electronics Engineering, Sri Krishna College of Engineering and Technology, Coimbatore, \\ 641008, India \\ ${ }^{3}$ School of Computing, Kalasalingam Academy of Research and Education, Krishnankoil, 626128, India \\ ${ }^{4}$ Department of Computer Science, College of Computer Engineering and Sciences, Prince Sattam Bin Abdulaziz University, \\ Alkharj, 11942, Saudi Arabia \\ ${ }^{5}$ Department of Applied Data Science, Noroff University College, Kristiansand, 4612, Norway \\ ${ }^{6}$ Department of ICT Convergence, Soonchunhyang University, Asan, 31538, Korea \\ ${ }^{*}$ Corresponding Author: Byeong-Gwon Kang. Email: bgkang@sch.ac.kr \\ Received: 10 August 2021; Accepted: 10 September 2021
}

\begin{abstract}
A mobile ad hoc network (MANET) involves a group of wireless mobile nodes which create an impermanent network with no central authority and infrastructure. The nodes in the MANET are highly mobile and it results in adequate network topology, link loss, and increase the re-initialization of the route discovery process. Route planning in MANET is a multi-hop communication process due to the restricted transmission range of the nodes. Location aided routing (LAR) is one of the effective routing protocols in MANET which suffers from the issue of high energy consumption. Though few research works have focused on resolving energy consumption problem in LAR, energy efficiency still remains a major design issue. In this aspect, this study introduces an energy aware metaheuristic optimization with LAR (EAMO-LAR) protocol for MANETs. The EAMO-LAR protocol makes use of manta ray foraging optimization algorithm (MRFO) to help the searching process for the individual solution to be passed to the LAR protocol. The fitness value of the created solutions is determined next to pass the solutions to the objective function. The MRFO algorithm is incorporated into the LAR protocol in the EAMO-LAR protocol to reduce the desired energy utilization. To ensure the improved routing efficiency of the proposed EAMO-LAR protocol, a series of simulations take place. The resultant experimental values pointed out the supreme outcome of the EAMO-LAR protocol over the recently compared methods. The resultant values demonstrated that the EAMO-LAR protocol has accomplished effectual results over the other existing techniques.
\end{abstract}

Keywords: MANET; routing; lar protocol; energy efficiency; mrfo algorithm; metaheuristics 


\section{Introduction}

An adhoc network belongs to a single session connection which doesn't utilize wireless routers/base stations and mainly it is made for transitory network connection. In these kinds of network, each node takes part in the routing actions without using any infrastructures. In place of classical routing, a flooding approach is used to the networks for transiting data [1]. Mainly, adhoc networks are utilized for crisis situations including military operations and natural disasters. The MANET is a multihop, dynamic topological mobile network which carries out autonomous operation offers routing service all over the nodes [2]. The node has constrained computation ability also capable of transmitting the data packet from source to the essential destination. These types of networks are suitable for distinct applications including emergency situations, outdoor activities, certain military operations, natural calamities, and communication in place with no wireless services.

MANET is an autonomous operation of mobile nodes using routing abilities interconnected with wireless connections [3]. All mobile nodes could perform as a router, and it is capable of communicating directly with other nodes in its physical neighbourhood. MANET is a group of mobile nodes which could vigorously alter location to create a network for exchanging data. Fig. 1 illustrates the structure of MANET. The key characteristics of MANET are automated self-maintenance, inexpensive deployment, self-configuring, and much needed for centralized administration or fixed network infrastructures. Because of its flexible nature, MANET is highly relevant in disaster relief, battlefield communications, educational, and commercial, emergency, search and rescue operations.

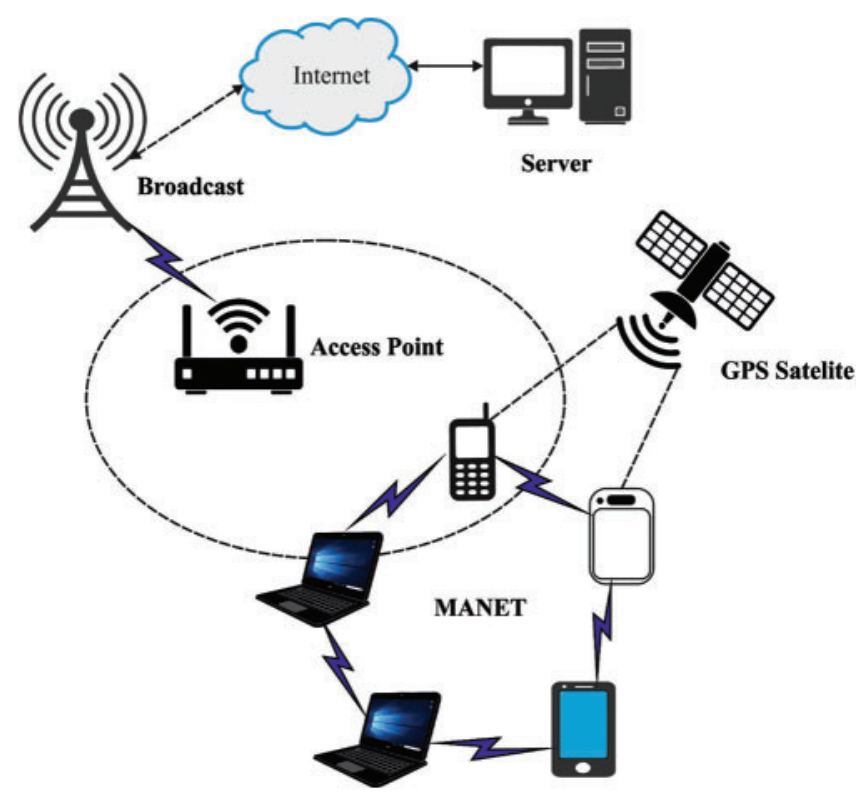

Figure 1: MANET architecture

The key challenge in MANETs is the development of dynamic routing protocol which could be aware of mobility and effective for determining the routes among the transmitting nodes with less overhead and better performance [4]. The routing protocol in MANET is classified into 3 classes according to how routing information is maintained and acquired using mobile nodes. 
The initial class refers to proactive (table driven) routing protocol. In such routing protocols, mobile node evaluates routes for each accessible node an analytic and preserve consistency and latest routing data with a periodic route upgrade procedure. An example of proactive routing protocol is optimized link state routing protocol (OLSR) and destination sequenced distance vector routing (DSDV). The next class of routing protocols is known as reactive (on-demand) routing protocol [5]. In this protocol, while any nodes want to interact with another node, it employs on-demand route discovery system to create links. Example of reactive routing protocol is AODV and dynamic source routing (DSR). A hybrid routing protocol is the 3rd class of MANET routing protocol. Hybrid routing protocol combines the optimal practices of reactive and proactive routing protocol. The zone routing protocol (ZRP) is an instance of hybrid routing protocol.

Location Aided Routing (LAR) protocols are a methodology which reduces overhead of route discovery with the help of Position details of mobile host. These position details might be attained by the global positioning system (GPS) [6]. LAR utilizes 2 flooding areas, the expected and forwarded regions. LAR protocols use position details for reducing the search area for a desirable path. Constraining the search area result in fewer route discovery messages [7]. If source nodes want to transmit data packets to destinations, first the source nodes must acquire the location of the destination mobile node by communicating position services i.e., in charge of mobile node position. This creates tracking and connection issues [8].

This study introduces an energy aware metaheuristic optimization with LAR (EAMO-LAR) protocol for MANETs. The EAMO-LAR protocol makes use of manta ray foraging optimization algorithm (MRFO) to help the searching process for the individual solution to be passed to the LAR protocol. The fitness value of the created solutions is determined next to pass the solutions to the objective function. The MRFO algorithm is incorporated into the LAR protocol in the EAMO-LAR protocol to reduce the desired energy utilization. To ensure the improved routing efficiency of the proposed EAMO-LAR protocol, a series of simulations take place.

In short, the key contributions of the paper are given as follows.

- Propose a novel EAMO-LAR protocol for energy efficient routing in MANET.

- Design a MFRO algorithm to aid the searching process for the individual solution to be passed to the LAR protocol.

- Derive a fitness value of the created solutions to pass the solutions to the objective function.

- Validate the performance of the EAMO-LAR protocol to ensure the improved routing efficiency interms of different measures.

The rest of the study is planned as follows. Section 2 briefs the existing routing techniques designed for MANET. Then, Section 3 introduces the proposed model and Section 4 provides the experimental validation. Lastly, Section 5 concludes the study.

\section{Related Works}

This section provides a detailed survey of available routing techniques in MANET to accomplish energy efficiency. Alsaqour et al. [9] proposed an approach named GALAR for enhancing the MANET routing protocol efficacy. The GALAR approach preserves an adoptive upgrade of the node position details by including the transferring node position details to the routing packet and select the transferring node to transmit the packets to their destination. The GALAR was created on the basis of a genetic optimization system which considers each participating factor in the delivery behaviour with criterion function optimization. In Hemalatha et al. [10], the optimal loop free route is developed using EO by FL approach and it guarantees increasing continuity. Firstly, 
in route exploration stage the EO approach using FL algorithm is utilized for establishing the optimum routing for transmitting the packets. For the applicable node election to route, levy flight distribution approach is utilized in this study. In route maintenance stage, calculate the position of the node previous to the data transmission. In route recovery stage or backward procedure, the backward response from the destinations is made. In the 4th stage, reactive path setup procedure is used. Lastly in multi-path procedure, the multi-path setup processes are used to the header through the intermediate node.

Agbon et al. [11] introduces the growth of a Throughput and Lifetime efficient routing protocol T-AODV for energy aware routing in MANET. TL-AODV is a development on the present E-AODV approach. Method: The challenge of electing energy effective route with constrained bottlenecks at intermediate nodes was modeled as the knapsack optimization issue. Depending on this issue, all nodes in the provided network calculate their CS state. In Abdali et al. [12], EALAR is an effective reactive MANET routing protocol i.e., attained by incorporating PSO using mutation operations to the traditional LAR protocol. But, the mutation operations (non-uniform) utilized in EALAR have few disadvantages that create EALAR to offer diversity of solutions, insufficient exploitation, and exploration. Hence, the aim is to develop an OPSO approach by adapting mutation operations (uniform) rather than non-uniform. The OPSO is incorporated into the LAR protocols for enhancing each crucial efficiency metric.

In Jubair et al. [13], a novel BOLSR protocol is presented for improving the energy utilization of the OLSR protocols. The symmetry among OLSRs of BA and MANET is they utilize similar methods to find the route by receiving and sending certain signal. The symmetry results in BOLSR protocols which defines the enhanced paths from source to destination nodes based on the energy dynamics of the node. Mukhedkar et al. [14] presents AES-TDCO according to the DCO approach, i.e., an energy and trust aware routing protocol. The presented DCO is involved in the optimum route selections depending on the modeled objective function according to trust factor, direct and indirect trusts, historical trust, recent trust, distance, delay, and link lifespan. The DCO approach is the incorporation of Dolphin Echolocation and CSO approach inherit fast global convergence. Zhang et al. [15] proposed a GA-BFO approach for performing the election of the optimum routing. Afterward finding multiple paths to the end nodes, the routes are started after the GA approaches are initialized. This approach rapidly find the position of maximal likelihood optimum path, i.e., the primary position of bacteria for the BFO approach. With the help of BFO approach, it is easier for searching for the highest value and the optimum paths for compensating for the weak accuracy of GA approach.

Sarhan et al. [16] proposed energy effective routing protocol that depends on the familiar AOMDV routing protocol and a bioinspired approach named EHO method. In the presented EHO-AOMDV approach the total energy spent by the node is optimized by categorizing nodes into 2 groups when paths are found out from the class of appropriate nodes with adequate energy for communication to decrease the likelihood of paths failure and the growing amount of dead nodes via highest data load. Taha et al. [17] highlight the energy usage in MANET with the help of FF algorithm for optimizing the energy utilization in AOMDV routing protocols. The presented protocols are called FF-AOMDV. The FF is utilized for finding the optimum paths from the source to destination nodes for reducing the energy utilization in multi-path routing.

\section{The Proposed Model}

In this study, a new EAMO-LAR protocol is designed for MANETs. The EAMO-LAR protocol makes use of MRFO to help the searching process for the individual solution to be 
passed to the LAR protocol. The fitness value of the created solutions is determined next to pass the solutions to the objective function. The MRFO algorithm is incorporated into the LAR protocol in the EAMO-LAR protocol to reduce the desired energy utilization.

\subsection{Overview of LAR}

LAR is presented by Ko et al. [18] as most primary routing protocols which regraded place data in routing in MANET. The LAR is dependent upon the source node (S) and depends on the place data of destination node (D). The predictable zone in this function is evaluated function is calculated as destination area for $\mathrm{D}$. The predictable region with $\mathrm{t}$ 1 being the $\mathrm{S}$ velocity of $\mathrm{D}$ at $\mathrm{L} 0$ ( $\mathrm{X} 0, \mathrm{Y} 0)$ within time $\mathrm{t} 0$ but $\mathrm{V}$ implies the mean velocity of $\mathrm{D}$. Therefore, the radius, $\mathrm{V}$ $(\mathrm{t} 1-\mathrm{t} 0)$ represents the area in mid-point of $\mathrm{L} 0(\mathrm{X} 0, \mathrm{Y} 0)$ to predictable zone. In LAR flood the network with RREQ, ensuring that packet is neither attaining the D node nor improving the rate of packet tranmitting. In order to LAR for working appropriately, the node is aware of node in the request region; so, the node is also preserved forward the flooded packet or drop it.

\subsection{Process Involved in MRFO Algorithm}

In this method, MR is determined as unique species although it appears to be terrific. Also, it is called a marine species. The summary of MR is a pair of pectoral fins and flat body that act as a tool for swimming as the bird flies in nature. A pair of cephalic lobe also existing in front of the terminal, giant mouths. With no sharp teeth, MR feeds plankton of small animals in the ocean. In foraging, they funnel water and prey to mouths via horn made cephalic lobes [19]. Then, prey could recognize from water using modified gill rakers. MR is classified into 2 species. At first, reef MR (manta alfredi) residing in the Indian Ocean, south and western Pacific i.e., suitable for attaining $5.5 \mathrm{~m}$ in width. Next, giant MR (manta birostris) was recognized using subtropical, tropical, and warm temperate ocean reaches $7 \mathrm{~m}$ in width. The stimulating fact about MR is that the maximal lifespan is twenty years; however, it doesn't live the actual lifespan because of external reasons and various factors.

The ocean is a main source of plankton. Hence, plankton isn't existing in certain regions i.e., manufactured by flow and ebb of tides. Eventually, MR is very effective in recognizing dense plankton. Unfortunately, the drawbacks regarding the MR is, foraging nature, and it moves by itself or in a collection of fifty; but, foraging is greatly observed in communities. When huge MR invokes foraging, it creates a line which follows in sequence. The small male MR is piggybacked on female one and move toward the top of back to map the beat of females' pectoral fins. Finally, plankton wasn't present it would be taken up by alternatives.

Next, the foraging principles are developed from cyclone foraging. The tails result connect with heads in a spiral for producing a spiraling vertex as a cyclonic form and attained water flows with a surface. When MR discovers a food source, it performed a series of back Somersault, where circles are made nearby the plankton for drawing it toward MR. Somersault is determined as a frequent, arbitrary, cyclical, and local motion which assist MR to consume better food source. Although the foraging nature is random, it is very effective. It is modeled mathematically and positions a new metaheuristic method called MRFO for computing global optimization.

MRFO was developed by 3 foraging behaviors such as somersault, chain, and cyclone foraging. The mathematical models are determined as follows. Fig. 2 demonstrates the search ability of MRFO technique. 


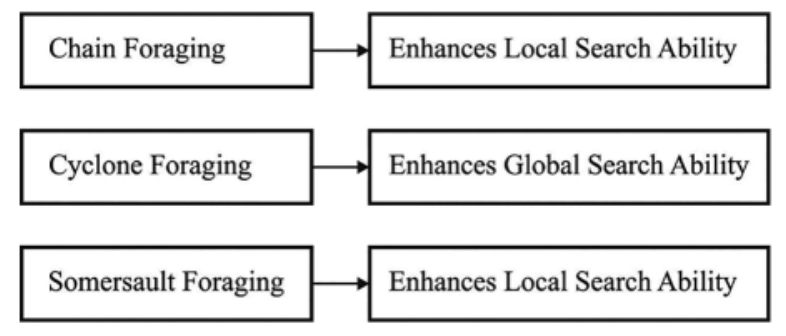

Figure 2: Search ability of MRFO

\section{Chain foraging}

In MRFO, MR can able to monitor the location of plankton and move toward it. When the location of plankton is larger, it is deliberated as an optimum one. Although the optimal solution is a dark room, MRFO considers that best solution as plankton using larger MR will attain an optimal food source. An individual with no initial moves toward food isn't worked; but, it has developed from them. Therefore, an individual is updated using the best solution i.e., recognized in front of them. The arithmetical approach of chain foraging is shown in the following:

$x_{i}^{d}(t+1)=\left\{\begin{array}{l}x_{i}^{d}(t)+r \cdot\left(x_{\text {best }}^{d}(t)-x_{i}^{d}(t)\right)+\alpha \cdot\left(x_{\text {best }}^{d}(t)-x_{i}^{d}(t)\right) i=1 \\ x_{i}^{d}(t)+r \cdot\left(x_{i-1}^{d}(t)-x_{i}^{d}(t)\right)+\alpha \cdot\left(x_{\text {best }}^{d}(t)-x_{i}^{d}(t)\right) i=2, \ldots, N\end{array}\right.$

$\alpha=2 \cdot r \cdot \sqrt{|\log (r)|}$

Whereas, $x_{i}^{d}(t)$ denotes the location of $i$ th individual at time $t$ in $d t$ dimension, $r$ denotes an arbitrary number in the range of zero and one, $a$ defines a weight coefficient, $x_{\text {best }}^{d}(t)$ represent the plankton with greater attention. The location upgrade of ith individual is $i$ th individual is determined with the location $x_{i-1}(t)$ of the $(i-1)$ th present individual and location $x_{\text {best }}(t)$ of the food.

\section{Cyclone foraging}

If a set of MR discovers dense plankton in marine water, it improves a longer foraging chain and moves toward the food in a spiral way. It is similar to the spiral foraging principles i.e., recognized in WOA. However, in the event of cyclone foraging method of MR, spiral movement for MRs swim in front of them. It follows a point in front of them and moves toward food using a spiral path. The arithmetical function of spiral formed motion of MR in two dimensional space is given below:

$$
\left\{\begin{array}{l}
X_{j}(t+1)=X_{\text {best }}+r \cdot\left(X_{i-1}(t)-X_{i}(t)\right)+e^{b w} \cdot \cos (2 \pi w) \cdot\left(X_{\text {best }}-X_{i}(t)\right) \\
Y_{i}(t+1)=Y_{\text {best }}+r \cdot\left(Y_{i-1}(t)-Y_{i}(t)\right)+e^{b w} \cdot \sin (2 \pi w) \cdot\left(Y_{\text {best }}-Y_{i}(t)\right)
\end{array}\right.
$$

Whereas $w$ represents an arbitrary number in zero and one.

The movement behaviors are transferred to n-D space. For easiness, the arithmetical method of cyclone foraging is shown in the following:

$x_{i}^{d}(t+1)=\left\{\begin{array}{l}x_{\text {best }}^{d}+r \cdot\left(x_{\text {best }}^{d}(t)-x_{i}^{d}(t)\right)+\beta \cdot\left(x_{\text {best }}^{d}(t)-x_{i}^{d}(t)\right) i=1 \\ x_{\text {best }}^{d}+r \cdot\left(x_{i-1}^{d}(t)-x_{i}^{d}(t)\right)+\beta \cdot\left(x_{\text {best }}^{d}(t)-x_{i}^{d}(t)\right) i=2, \ldots, N\end{array}\right.$ 
$\beta=2 e^{r_{1} \frac{T-t+1}{T}} \cdot \sin \left(2 \pi r_{1}\right)$

where as $\beta$ indicates the weight coefficient, $T$ denotes high amounts of iteration, and $r_{1}$ represents rand value from zero and one. The individual performs an exploration based on food as the reference location; therefore the cyclone foraging has improved exploitation for regions with an optimal solution as recognized. Also, it is employed to enhance the searching procedure. It forces the individual for searching for a novel position from the recent and improved one by assigning a novel arbitrary location from comprehensive search space as the reference location. It very focusses on the searching procedure and activates MRFO for reaching the extreme global search in which the function is given as follows:

$x_{\text {rand }}^{d}=L b^{d}+r \cdot\left(U b^{d}-L b^{d}\right)$

$x_{i}^{d}(t+1)=\left\{\begin{array}{l}x_{\text {rand }}^{d}+r \cdot\left(x_{\text {rand }}^{d}-x_{i}^{d}(t)\right)+\beta \cdot\left(x_{\text {rand }}^{d}-x_{i}^{d}(t)\right) i=1 \\ x_{\text {rand }}^{d}+r \cdot\left(x_{i-l}^{d}(t)-x_{i}^{d}(t)\right)+\beta \cdot\left(x_{\text {rand }}^{d}-x_{i}^{d}(t)\right) i=2, \ldots, N\end{array}\right.$

Whereas $x_{\text {rand }}^{d}$ denotes the arbitrary location, i.e., produced from search space, $L b^{d}$ and $U b^{d}$ represents lower and upper limit of dth dimension, correspondingly.

\section{Somersault foraging}

Now, the location of food is stated as a pivot. Therefore, it updates the position nearby the best location establishes until now. The arithmetical method could be evolved in the following:

$x_{i}^{d}(t+1)=x_{i}^{d}(t)+S \cdot\left(r_{2} \cdot x_{\text {best }}^{d}-r_{3} \cdot x_{i}^{d}(t)\right), i=1, \ldots, N$

Whereas $S$ represents the somersault factors that select somersault rank and $S=2, r_{2}$ and $r_{3}$ indicates 2 random numbers in the range of zero and one.

From Eq. (8), and depiction of few results range, it is feasible for an individual to swim toward the location for searching an application located amongst symmetrical and current positions on an improved location. Fig. 3 illustrates the flowchart of MRFO technique.

\subsection{Algorithmic Design of EAMO-LAR Algorithm}

The presented technique of LAR protocol has been called EAMO-LAR that adjusts the optimization MRFO technique. The optimization technique supports exploring the space to the individual solutions that exist fed as to LAR simulators for the generation of network measure. Afterward, the fitness value of created solutions was computed then feed the solutions were to the objective function. The source nodes (blue) make the data packets and transmits the RREQ packets for finding routes nearby destination. A destination node (yellow nodes) are the nodes for that the data packets are delivered. An inter-mediate node (green nodes) hold the RREQ packets and for broadcasting to their neighbours. The baselines are a vectors that links the source node as well as destination node. The distance (x. destination) has been Euclidian distance amongst the node $x$ (intermediate node) and destination node. The angle $x$ has been angles amongst the vectors and baseline which links the source and node $x$ to destinations. Also, the present place of all nodes is recognized as network which continues an upgraded record of place and energy of all nodes and their neighbours. The place of destinations are source nodes; therefore, these places are further to place of source nodes, and the place of source nodes is further to the RREQ packets. 


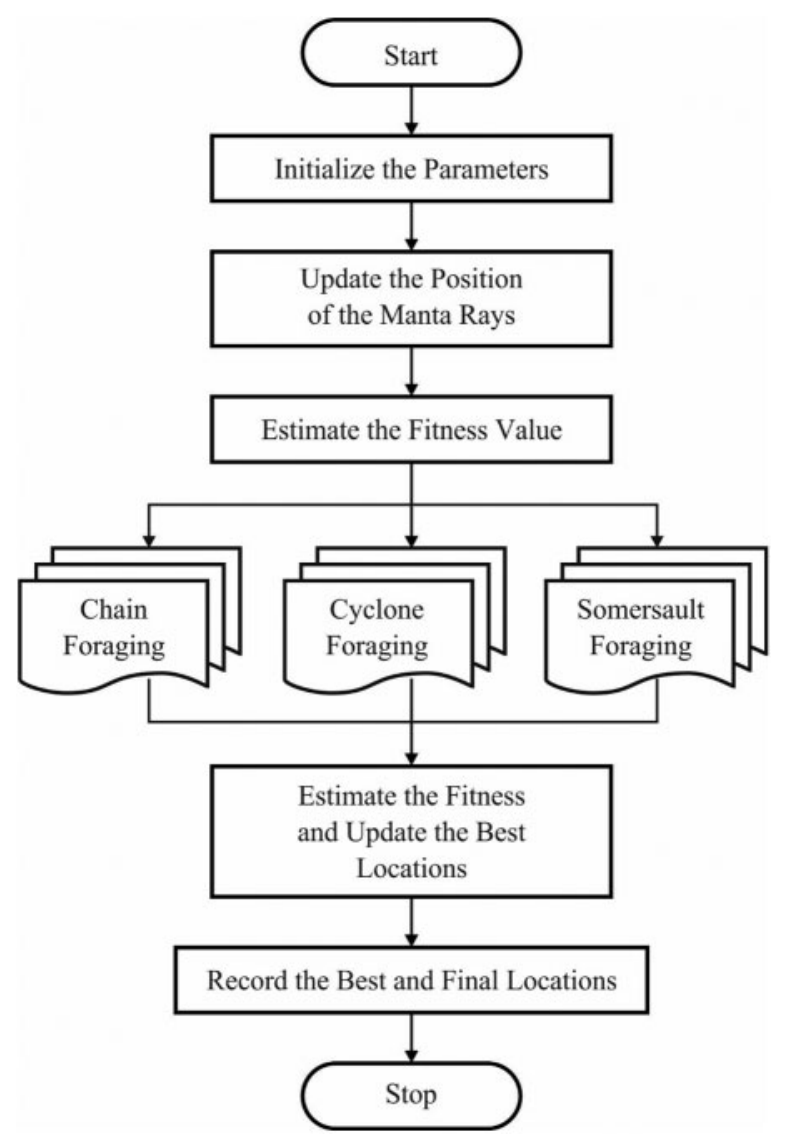

Figure 3: Flowchart of MRFO

A data guarantee in which all the nodes hold the RREQ packets from the network recognizes the places of source node and destination node [20]. Furthermore, the intermediate nodes were needed to happen in any situations are containing the energy level, angle, and distance that exists computed in the condition functions utilizing the subsequent equation:

CriterionFunction $=f_{\text {node }}=w_{1} *$ Angle $($ node $)+w_{2}$

Dis $($ node $\times$.destination $)+w_{3} *$ Energyconsumption (node $)$

where

Angle $($ node $)=\frac{\text { Angle }(\text { node })}{180} \in[0,1]$

Distance $($ node $\times$. destination $)=\frac{\text { Dis }(\text { node } \times \text {.destination })}{\text { lengthofenviroment }{ }^{\text {sdiagonal }}} \in[0,1]$

Energyconsumption $($ node $)=\frac{\text { InitialBattery }(\text { node })-\text { CurrentBattery }(\text { node })}{\text { InitialBattery }(\text { node })} \in[0,1]$ 
$w_{1}, w_{2}$, and $w_{3} \in[01]$ implies the weighting factor of parameters are utilized from the objective function and show a part in the efficiency of request region. Then, computing the 3 situations, the FR resolves the several nodes forward RREQ.

Considering, it is 3 intermediate nodes $(A, B, C)$ wait to forward the RREQ to next node, all the nodes are needed for presenting 3 situations such as the angle, distance, and energy level that exists computed as condition function. When the node $A$ is lesser angle, distance, and maximum energy level and $A$ has been superior to $B$ and $C$, afterward, the nodes are arranged in ascending sequence. The values of $A$ regards as threshold. When the $n$ node is superior to $A$, the threshold is ended and changed with node $n$.

An optimization technique helps in exploring the search space to individual solutions that exist feed as to LAR simulators for generation of network measure. Afterward, the fitness value of created solutions are estimated then feed the solution as to objective function. The computation of the fitness value of created solution also attain predictable result, the convenient value for weights $w_{1}, w_{2}$, and $w_{3}$ are elected. Also, the FR is contained in the manta ray (MR) place. Besides, CZR has been valuing $\in[0.1]$ which defines the coverage area radius then presence multiplied with $\max$ coverage area. The CZR has been contained in MR place by utilizing Eq. (13):

$F\left(n_{i}\right)=w_{1} \theta_{i}+w_{2} d_{i}+w_{3} e_{i}$

where $\theta$ implies the angle of subject nodes $i$ in concern of baselines amongst the destination and source; $d$ refer the distances amongst $i$ and destination; $e$ refers the existing energy of $i$, in which $w_{1}, w_{2}$, and $w_{3}$ signify the weighting factor of variables which are utilized from the objective function.

\section{Performance Validation}

This section investigates the routing performance of the EAMO-LAR technique with existing techniques [12] interms of different aspects. Tab. 1 showcases the detailed comparative study of the EAMO-LAR technique interms of PDR, overhead, and ETE delay. From investigates the results analysis of the EAMO-LAR technique interms of overhead under dissimilar number of nodes. The figure exhibited that the EAMO-LAR technique has demonstrated improved outcomes with minimal overhead. For instance, with 50 nodes, the EAMO-LAR technique has obtained a reduced overhead of 18.24 whereas the DLAR, EALAR, and OPSO-LAR techniques have attained a raised overhead of 387.31, 71.56, and 20.22 respectively. Simultaneously, with 70 nodes, the EAMO-LAR manner has gained a lesser overhead of 25.14 whereas the DLAR, EALAR, and OPSO-LAR techniques have reached a higher overhead of 271.08, 64.72, and 25.07 respectively. Concurrently, with 100 nodes, the EAMO-LAR technique has obtained a minimum overhead of 15.32 whereas the DLAR, EALAR, and OPSO-LAR methodologies have achieved an increased overhead of $232.4,58.97$, and 17.32 correspondingly.

Fig. 4 exhibits the PDR analysis of the EAMO-LAR technique with existing approaches under diverse node counts. The figure demonstrated that the EAMO-LAR technique has accomplished significant performance with the increased PDR. For instance, with 50 nodes, the EAMOLAR technique has resulted in a higher PDR of $41.17 \%$ whereas the DLAR, EALAR, and OPSO-LAR techniques have led to a lower PDR of $35.14 \%, 29.07 \%$, and $25.60 \%$ respectively. Also, with 70 nodes, the EAMO-LAR methodology has resulted in an increased PDR of $66.39 \%$ whereas the DLAR, EALAR, and OPSO-LAR manners have led to a lesser PDR of 39.92\%, $53.36 \%$, and $60.31 \%$ correspondingly. At last, with 100 nodes, the EAMO-LAR approach has 
resulted in a superior PDR of $95.85 \%$ whereas the DLAR, EALAR, and OPSO-LAR methods have led to a minimal PDR of $44.90 \%, 74.62 \%$, and $89.81 \%$ correspondingly.

Table 1: Result analysis of EAMO-LAR model with different measures under number of nodes

\begin{tabular}{|c|c|c|c|c|}
\hline \multicolumn{5}{|l|}{ PDR (\%) } \\
\hline No. of nodes & DLAR & EALAR & OPSO-LAR & EAMO-LAR \\
\hline 50 & 35.14 & 29.07 & 25.60 & 41.17 \\
\hline 60 & 36.66 & 42.30 & 45.56 & 51.64 \\
\hline 70 & 39.92 & 53.36 & 60.31 & 66.39 \\
\hline 80 & 37.53 & 57.49 & 67.46 & 72.55 \\
\hline 90 & 43.82 & 70.07 & 70.28 & 75.33 \\
\hline 100 & 44.90 & 74.62 & 89.81 & 95.85 \\
\hline \multicolumn{5}{|l|}{ Overhead } \\
\hline No. of nodes & DLAR & EALAR & OPSO-LAR & EAMO-LAR \\
\hline 50 & 387.31 & 71.56 & 20.22 & 18.24 \\
\hline 60 & 342.76 & 66.72 & 7.63 & 5.66 \\
\hline 70 & 271.08 & 64.78 & 25.07 & 25.14 \\
\hline 80 & 292.39 & 68.65 & 21.19 & 19.29 \\
\hline 90 & 272.05 & 58.00 & 20.22 & 19.24 \\
\hline 100 & 232.34 & 58.97 & 17.32 & 15.32 \\
\hline \multicolumn{5}{|c|}{ End to end delay (sec) } \\
\hline No. of nodes & DLAR & EALAR & OPSO-LAR & EAMO-LAR \\
\hline 50 & 2.74 & 4.46 & 5.15 & 7.23 \\
\hline 60 & 3.29 & 3.99 & 4.74 & 5.78 \\
\hline 70 & 2.99 & 3.67 & 4.28 & 4.86 \\
\hline 80 & 3.25 & 3.52 & 3.65 & 5.73 \\
\hline 90 & 2.67 & 3.00 & 3.41 & 4.42 \\
\hline 100 & 2.86 & 2.91 & 2.94 & 3.43 \\
\hline
\end{tabular}

Fig. 5 displays the ETED analysis of the EAMO-LAR technique with existing approaches under varied node count. The figure showcased that the EAMO-LAR manner has accomplished important performance with the improved ETED. For instance, with 50 nodes, the EAMO-LAR algorithm has resulted in a superior ETED of $7.23 \mathrm{~s}$ whereas the DLAR, EALAR, and OPSOLAR methods have led to a minimal ETED of $2.74 \mathrm{~s}, 4.46 \mathrm{~s}$, and $5.15 \mathrm{~s}$ correspondingly. In addition, with 70 nodes, the EAMO-LAR methodology has resulted in an improved ETED of $4.86 \mathrm{~s}$ whereas the DLAR, EALAR, and OPSO-LAR approaches have led to the least ETED of $2.99 \mathrm{~s}, 3.67 \mathrm{~s}$, and $4.28 \mathrm{~s}$ correspondingly. Finally, with 100 nodes, the EAMO-LAR algorithm has resulted in an increased ETED of $3.43 \mathrm{~s}$ whereas the DLAR, EALAR, and OPSO-LAR methods have led to a decreased ETED of $2.86 \mathrm{~s}, 2.91 \mathrm{~s}$, and $2.94 \mathrm{~s}$ correspondingly.

Tab. 2 explores the results analysis of the EAMO-LAR algorithm interms of EPP in dissimilar number of nodes. The figure outperformed that the EAMO-LAR method has showcased 
enhanced results with the lower EPP. For sample, with 50 nodes, the EAMO-LAR manner has reached a minimal EPP of 6.44 whereas the DLAR, EALAR, and OPSO-LAR algorithms have attained a raised EPP of 7.39, 7.79, and 7.98 respectively. Also, with 70 nodes, the EAMOLAR approach has reached a lesser EPP of 2.65 whereas the DLAR, EALAR, and OPSO-LAR techniques have obtained a maximum EPP of 4.45, 2.80, and 2.59 correspondingly. Concurrently, with 100 nodes, the EAMO-LAR manner has reached the least EPP of 0.76 whereas the DLAR, EALAR, and OPSO-LAR algorithms have gained an improved EPP of 2.86, 1.51, and 0.90 correspondingly.

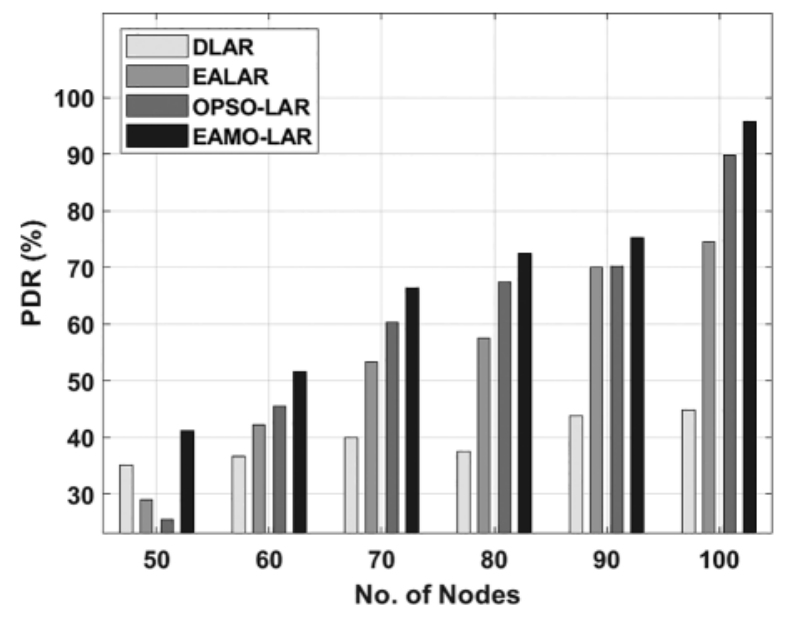

Figure 4: PDR analysis of EAMO-LAR model under different nodes

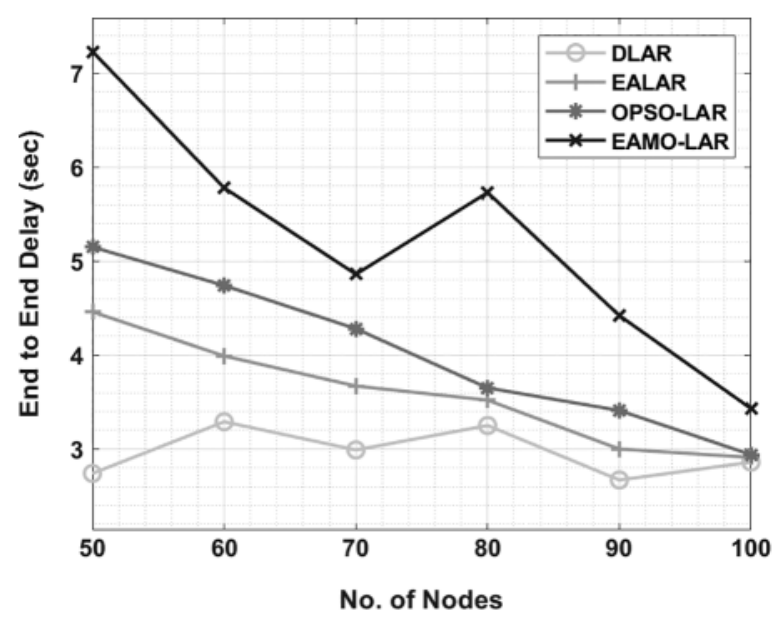

Figure 5: ETED analysis of EAMO-LAR model under different nodes 
Table 2: Energy per packet analysis of EAMO-LAR model

\begin{tabular}{lllll}
\hline Energy per packet (EPP) (energy unit) & & & \\
\hline No. of nodes & DLAR & EALAR & OPSO-LAR & EAMO-LAR \\
\hline 50 & 7.39 & 7.79 & 7.98 & 6.44 \\
60 & 5.69 & 4.29 & 3.53 & 1.53 \\
70 & 4.45 & 2.80 & 2.59 & 2.65 \\
80 & 4.06 & 2.35 & 1.35 & 0.45 \\
90 & 3.24 & 1.76 & 1.78 & 1.20 \\
100 & 2.86 & 1.51 & 0.90 & 0.76 \\
\hline
\end{tabular}

Tab. 3 and Fig. 6 examines the outcomes analysis of the EAMO-LAR approach with respect to NRL under dissimilar number of speeds. The figure demonstrated that the EAMO-LAR manner has exhibited increased outcomes with the lesser NRL. For instance, with 10 speeds, the EAMO-LAR method has reached a minimum NRL of 3.32 whereas the DLAR, EALAR, and OPSO-LAR algorithms have gained an increased NRL of 4.37, 3.81, and 3.97 correspondingly. Concurrently, with 30 speeds, the EAMO-LAR method has achieved a lesser NRL of 4.39 whereas the DLAR, EALAR, and OPSO-LAR techniques have attained a raised NRL of $6.80,5.06$, and 5.24 correspondingly. Concurrently, with 50 speeds, the EAMO-LAR manner has obtained a minimal NRL of 4.35 whereas the DLAR, EALAR, and OPSO-LAR methods have attained an improved NRL of 5.95, 5.52, and 5.02 respectively.

Table 3: NRL analysis of EAMO-LAR model with existing techniques

\begin{tabular}{lllll}
\hline \multicolumn{2}{l}{ Normalized routing load $(\mathrm{NRL})$} & & & \\
\hline Speed $(\mathrm{ms})$ & DLAR & EALAR & OPSO-LAR & EAMO-LAR \\
\hline 10 & 4.37 & 3.81 & 3.97 & 3.32 \\
20 & 6.47 & 4.31 & 3.99 & 3.56 \\
30 & 6.80 & 5.06 & 5.24 & 4.39 \\
40 & 5.62 & 4.57 & 4.11 & 3.71 \\
50 & 5.95 & 5.52 & 5.02 & 4.35 \\
\hline
\end{tabular}

From the above mentioned tables and figures, it is apparent that the EMAO-LAR technique has reduced the energy consumption of the conventional LAR protocol. Besides, the use of fitness function helps to optimally select the solutions for the objective function and also accomplished effective routing process. Therefore, the EAMO-LAR technique can be utilized as an effective routing tool for MANET. 


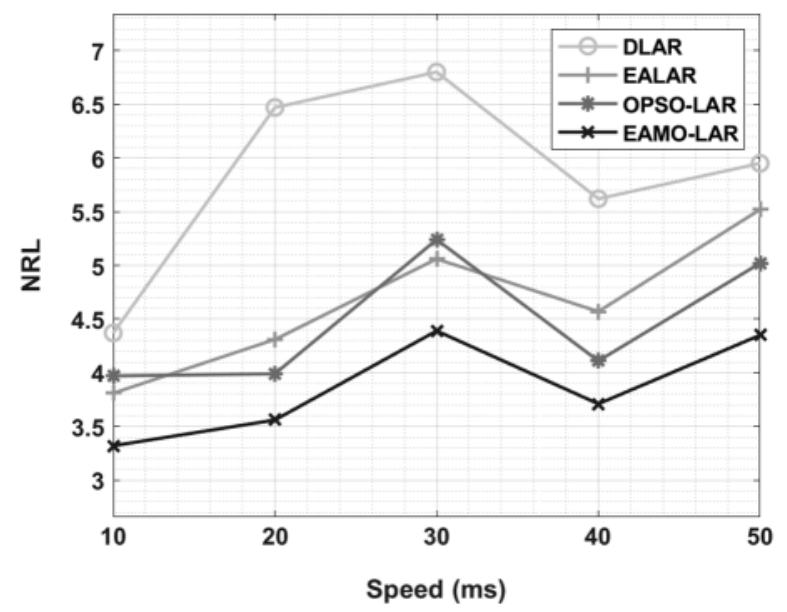

Figure 6: NRL analysis of EAMO-LAR model with existing techniques

\section{Conclusion}

In this study, a new EAMO-LAR protocol is designed for MANETs to achieve energy efficient route selection process. The EAMO-LAR protocol makes use of MRFO to help the searching process for the individual solution to be passed to the LAR protocol. The fitness value of the created solutions is determined next to pass the solutions to the objective function. The MRFO algorithm is incorporated into the LAR protocol in the EAMO-LAR protocol to reduce the desired energy utilization. To ensure the improved routing efficiency of the proposed EAMO-LAR protocol, a series of simulations take place. The resultant experimental values pointed out the supreme outcome of the EAMO-LAR protocol over the recent compared methods. The resultant values demonstrated that the EAMO-LAR protocol has accomplished effectual results over the other existing techniques. In future, effective node localization and time synchronization techniques can be designed for MANETs.

Funding Statement: This research was supported by the MSIT (Ministry of Science and ICT), Korea, under the ICAN (ICT Challenge and Advanced Network of HRD) program (IITP-20212020-0-01832) supervised by the IITP (Institute of Information \& Communications Technology Planning \& Evaluation) and the Soonchunhyang University Research Fund.

Conflicts of Interest: The authors declare that they have no conflicts of interest to report regarding the present study.

\section{References}

[1] C. Z. Sirmollo and M. A. Bitew, "Mobility-aware routing algorithm for mobile ad hoc networks," Wireless Communications and Mobile Computing, vol. 2021, pp. 1-12, 2021.

[2] D. Gupta, A. Khanna, S. K. Lakshmanaprabu, K. Shankar, V. Furtado et al., "Efficient artificial fish swarm based clustering approach on mobility aware energy-efficient for MANET," Transactions on Emerging Telecommunications Technologies, vol. 30, no. 9, pp. 1-10, 2019.

[3] B. Bhushan, S. Gupta and C. K. Nagpal, "Comparison of on demand routing protocols," International Journal of Information Technology and Computer Science (IJITCS), vol. 5, no. 3, pp. 61-68, 2013. 
[4] M. Elhoseny, R. S. Rajan, M. Hammoudeh, K. Shankar and O. Aldabbas, "Swarm intelligencebased energy efficient clustering with multihop routing protocol for sustainable wireless sensor networks," International Journal of Distributed Sensor Networks, vol. 16, no. 9, pp. 155014772094913 , 2020.

[5] C. Yu, B. Lee and H. Y. Youn, "Energy efficient routing protocols for mobile ad hoc networks," Wireless Communications and Mobile Computing, vol. 3, no. 8, pp. 959-973, 2003.

[6] A. K. Dutta, M. Elhoseny, V. Dahiya and K. Shankar, "An efficient hierarchical clustering protocol for multihop internet of vehicles communication," Transactions on Emerging Telecommunications Technologies, vol. 31, no. 5, pp. 1-11, 2020.

[7] T. Vaiyapuri, V. S. Parvathy, V. Manikandan, N. Krishnaraj, D. Gupta et al., "A novel hybrid optimization for cluster-based routing protocol in information-centric wireless sensor networks for iot based mobile edge computing," Wireless Personal Communications, vol. 121, pp. 1-24, 2021.

[8] P. S. Raman, K. Shankar and M. Ilayaraja, "Securing cluster based routing against cooperative black hole attack in mobile ad hoc network," International Journal of Engineering and Technology (UAE), vol. 7, no. 1.9, pp. 6, 2018.

[9] R. Alsaqour, S. Kamal, M. Abdelhaq and Y. A. Jeroudi, "Genetic algorithm routing protocol for mobile ad hoc network," Computers, Materials \& Continua, vol. 68, no. 1, pp. 941-960, 2021.

[10] R. Hemalatha, R. Umamaheswari and S. Jothi, "LF Distribution and Equilibrium Optimizer Based Fuzzy Logic for Multipath Routing in MANET,"Wireless Personal Communications, vol. 120, no. 2, pp. 1837-1861, 2021. http://dx.doi.org/10.1007/s11277-021-08537-6.

[11] E. E. Agbon, M. G. Awe, H. I. Yarima, F. C. Njoku and I. Rahman, "Optimization approach for throughput and lifetime maximization of energy aware manet," Computing \& Information Systems, vol. 23, no. 1, pp. 9-18, 2019.

[12] T. A. N. Abdali, R. Hassan, R. C. Muniyandi, A. H. M. Aman, Q. N. Nguyen et al., "Optimized particle swarm optimization algorithm for the realization of an enhanced energy-aware location-aided routing protocol in manet," Information, vol. 11, no. 11, pp. 529, 2020.

[13] M. A. Jubair, S. A. Mostafa, R. C. Muniyandi, H. Mahdin, A. Mustapha et al., "Bat optimized link state routing protocol for energy-aware mobile ad-hoc networks," Symmetry, vol. 11, no. 11, pp. 1409, 2019.

[14] M. Mukhedkar and U. Kolekar, "Trust-based secure routing in mobile ad hoc network using hybrid optimization algorithm," The Computer Journal, vol. 62, no. 10, pp. 1528-1545, 2019.

[15] D. Zhang, S. Liu, X. Liu, T. Zhang and Y. Cui, "Novel dynamic source routing protocol (DSR) based on genetic algorithm-bacterial foraging optimization (GA-BFO)," International Journal of Communication Systems, vol. 31, no. 18, pp. e3824, 2018.

[16] S. Sarhan and S. Sarhan, "Elephant herding optimization ad hoc on-demand multipath distance vector routing protocol for manet," IEEE Access, vol. 9, pp. 39489-39499, 2021.

[17] A. Taha, R. Alsaqour, M. Uddin, M. Abdelhaq and T. Saba, "Energy efficient multipath routing protocol for mobile ad-hoc network using the fitness function," IEEE Access, vol. 5, pp. 10369-10381, 2017.

[18] Y. B. Ko and N. H. Vaidya, "Anycasting-based protocol for geocast service in mobile ad hoc networks," Computer Networks, vol. 41, no. 6, pp. 743-760, 2003.

[19] M. G. Hemeida, A. A. Ibrahim, A. A. A. Mohamed, S. Alkhalaf and A. M. B. E. Dine, "Optimal allocation of distributed generators DG based manta Ray foraging optimization algorithm (MRFO)," Ain Shams Engineering Journal, vol. 12, no. 1, pp. 609-619, 2021.

[20] K. Ghosh, S. Roy and P. K. Das, "I-min: An intelligent fermat point based energy efficient geographic packet forwarding technique for wireless sensor and ad hoc networks," International Journal on Applications of Wireless Ad hoc Networks and Sensor Networks, vol. 2, no. 2, pp. 34 44, 2010. 